from the constraints provided by the disulphide bridges) only to the extent that it incorporates the correctly identified residues mentioned above juxtaposed in the active centre. For the rest, the non-covalent interactions deduced by Scheraga and co-workers are evidently almost wholly specious. Earlier models, by Saroff and by Scheraga, are as different from the above model as they are from the actual structure. At the present stage it therefore appears that the indirect approach to the construction of three-dimensional protein structures is of dubious value.

The appearance of more complete structural data will offer the possibility of interpreting the mechanism of nucleolytic hydrolysis, and will now be awaited with the greatest interest.

\section{Momentum Dependent Interactions}

\section{from a Correspondent in High-energy Physics}

Is a recent experiment at the Cornell $2 \mathrm{GeV}$ synchrotron, K. Kerkelman et al. (Physics Letters, 24B, 165; 1967) have measured the momentum dependence of the magnetic dipole transition between a proton and the positively charged $N^{*}$ resonance at $1,230 \mathrm{MeV}$.

The inelastic cross-sections for the processes in which an electron striking a proton produce in the final state an electron together with either a proton and a neutral pion or a neutron and a positively charged pion show a marked enhancement when the effective mass of the final nucleon and pion system is near $1,200 \mathrm{MeV}$. This effect is usually interpreted as a first order electromagnetic process in which the scattered electron emits a virtual photon-one for which the effective mass is not zero-which in turn excites the proton into a state with mass $1,200 \mathrm{MeV}$. A final pion and nucleon are then produced by the (very fast) strong decay of this $N^{*}$ resonance - or particle as it is frequently called. On this interpretation of the state as a particle with isotopic spin $3 / 2$, positive parity relative to the nucleon and spin $3 / 2$, the transition from the nucleon induced by the virtual photon can be shown by general covariance and angular momentum properties to be of only three possible types. These are a magnetic dipole $(M 1)$, an electric quadrupole $(E 2)$ and a Coulomb octupole $(C 2)$ transition, each of which is measured in terms of a corresponding form factor depending only on the momentum transfer (the effective invariant mass of the virtual photon).

If the analysis in terms of a virtual photon is valid, then these form factors have already been measured for zero momentum transfer by experiments in which the $N^{*}$ state is excited by a real photon (Gourdin, M., and Salin, P. H., Nuovo Cimento, 27, 193; 1963). Thus it is known that the form factor describing the $M 1$ transition has a value at zero momentum transfer of $3.00 \pm 0.01$ in units of nuclear Bohr magnetons and that the other two transitions are much smaller (less than 5 per cent). In the electro-production experiment the analysis is therefore based on the assumption that only the $M 1$ transition contributes-a fair assumption for this first experiment. This simplification makes it possible in principle to measure the $M 1$ transition form factor $G_{M}^{*}\left(k^{2}\right)$ by the standard techniques of observing only the scattered electron, provided that the dominant contribution is known to be from the $N^{*}$ and not from non-resonant background effects. The technical advance which has made this experiment possible is that events have been selected in which a final proton and neutral pion are detected in coincidence. Since the photoproduction results indicate that this final state (in contrast with the neutronpositive pion state) is predominantly resonant, the background is largely eliminated.

The results obtained over a range of momentum transfers up to $8(\mathrm{GeV})^{2}$ extrapolate neatly back to the value at zero momentum transfer measured in the photoproduction experiments, and thus confirm the validity of the model assumed. Furthermore, the momentum dependence is roughly in agreement with the predictions of both the dispersion theory and the relativistic symmetry schemes for the strong interactions which are presumed to be the source of this effect, although detailed comparison requires much improved experiments.

This experiment surely marks a new era of sophistication in the use of electron machines. The new synchrotrons in Germany (DESY) and England (NINA) are starting operation without any substantial energy advantage over earlier machines. Crude elastic electron scattering experiments can hardly justify extensive running time, and inelastic experiments, particularly of the coincidence and polarization types, must figure largely in their future programmes.

\section{Egon Bretscher Retires}

The Atomic Energy Research Establishment at Harwell, now 21 years old, is branching out into new fields with the setting up of centres for ceramics and materials testing. It remains, however, a place with powerful associations for physicists, and two of them have recently been writing of the retirement of Dr. Egon Bretscher, head of the Nuclear Physics Division since 1948, who had been associated with work on atomic energy since the early days of the war. After graduating as a chemist in 1927, Bretscher had moved to Cambridge, where he collaborated with Feather on the problem of uranium $Z$ when, according to Feather, "progress depended essentially on the trustworthiness of Bretscher's chemical preparations: time has shown that they were impeccable". This was followed by neutron bombardment with particles from the one million volt accelerator, and towards the end of 1938 the group was making preparations to study the $\mathrm{X}$ radiation of the supposed transuranic elements-those which, a few months later, were shown by Hahn and Strassman to be radioactive isotopes of elements with smaller atomic number.

A. P. French explains how Bretscher turned from the radiochemistry of uranium to the measurement of fission cross-sections for fast neutrons using neutrons from the $\mathrm{D}+\mathrm{D}$ reaction. One of Bretscher's innovations at this point, in 1942, was to devise a way of extending the technique of measurement with a homogeneous ionization chamber so as to measure neutron flux in the presence of a strong gamma-ray background as with neutrons from the ${ }^{12} \mathrm{C}+\mathrm{D}$ reaction. This work was helped along by the possession in Cambridge of $2 \mathrm{~g}$ of fully deuterated paraffin wax, prepared a few years earlier at the heavy water plant in Norway. French says that this was used to line a chamber that was filled with deutero-ethylene and used differ- 